CONCISE REPORT

\title{
Modification of hypertension and hypercholesterolaemia in patients with systemic lupus erythematosus: a quality improvement study
}

\author{
M B Urowitz, D D Gladman, D Ibanez, Y Berliner
}

Ann Rheum Dis 2006;65:115-117. doi: 10.1136/ard.2005.038802

Background: Hypercholesterolaemia and hypertension are risk factors for coronary artery disease in patients with systemic lupus erythematosus (SLE).

Objective: To examine the recognition and management of hypercholesterolaemia and hypertension in patients with SLE before and after a quality improvement study.

Method: Patients with SLE have been followed up prospectively at the University of Toronto Lupus Clinic since 1970. The charts of all patients who entered the clinic since 1990 were reviewed to ensure completeness of data on antihypertensive treatment (AHT) and lipid lowering agents (LLA). Recognition and management of hypercholesterolaemia and hypertension were evaluated for the periods 19901995 and 1996-2001.

Results: Comparison of treatment between time periods showed that during 1990-1995 204/559 (36\%) patients seen were hypertensive, of whom $180(88 \%)$ were receiving AHT, and during 1996-2001 241/576 (42\%) patients seen were hypertensive, of whom 232 (96\%) were receiving AHT $(p=0.0013)$. A comparison of treatment for hypercholesterolaemia showed that 21/236 (9\%) hypercholesterolaemic patients were being treated with LLA in the earlier period compared with $74 / 261(28 \%)$ in the later period $(p<0.0001)$.

Conclusions: Treatment for hypertension and hyperlipidaemia has increased in the past 6 years compared with the previous 6 years, but a number of patients eligible for these treatments remain untreated.

A ccelerated atherosclerosis in patients with systemic lupus erythematosus (SLE) leads to a high prevalence of coronary artery disease (CAD). ${ }^{1}$ Studies investigating the factors associated with CAD in SLE have identified both traditional risk factors for CAD, and factors related to disease and treatment. Specifically, three large prospective studies, conducted in the late eighties and early nineties, identified hypercholesterolaemia as a risk factor for CAD, with two of these studies also identifying hypertension as a risk factor. ${ }^{2-5}$ Additionally, carotid ultrasound studies have found that factors significantly associated with perfusion abnormalities include higher total cholesterol, higher low density lipoprotein cholesterol, higher blood pressure, and higher body mass index. ${ }^{6}$ Recently, Bruce et al found that hypertension, any occurrence of raised cholesterol, and the total cholesterol:low density lipoprotein cholesterol ratio were associated with abnormal dual isotope myocardial perfusion imaging study results. ${ }^{7}$

The recognition and management of hypercholesterolaemia and hypertension by physicians in patients with lupus represents an important aspect of the care afforded to these patients. In 1998 we reported a quality improvement study of patients with SLE followed up before $1996 .{ }^{\circ}$ We showed that in patients who subsequently developed acute coronary events, potentially reversible risk factors before the event were not well managed. The purpose of the current study was to compare the adequacy of management of hypercholesterolaemia and hypertension in all lupus patients subsequent to 1996 compared with those patients followed up before 1996 at the University of Toronto Lupus Clinic.

\section{METHODS}

Patients with lupus have been followed up prospectively at the clinic since 1970. Clinical and laboratory information is collected on a standard protocol and stored on a computer database. All patients are assessed by the directors of the clinic (MBU or DDG) or by a clinical fellow trained by them. The charts of all patients who entered the clinic since 1990 were reviewed, to ensure completeness of the data in the database for both antihypertensive treatment (AHT) and lipid lowering agents (LLA).

Hypertension was defined as blood pressure $>140 \mathrm{~mm} \mathrm{Hg}$ systolic and/or $>90 \mathrm{~mm} \mathrm{Hg}$ diastolic for at least 3 months, or receiving antihypertensive treatment. The prescription of antihypertensive drugs was considered appropriate management.

Hypercholesterolaemia was defined as random total cholesterol of $>6.2 \mathrm{mmol} / \mathrm{l}$, or treatment with an LLA. We used total cholesterol rather than lipid fractions because a fasting level is not required. The prescription of LLA was considered appropriate management.

Disease activity at each visit was measured using the SLE Disease Activity Index (SLEDAI) 2000. ${ }^{9}$ Disease activity over time was calculated using the adjusted mean SLEDAI. ${ }^{10}$ Active renal disease was defined by the presence of sterile pyuria and haematuria, proteinuria $>0.5 \mathrm{~g} / 24 \mathrm{~h}$, and casts. Nephrotic syndrome was defined by proteinuria $>3.5 \mathrm{~g} / 24 \mathrm{~h}$ and renal insufficiency was defined by an increased serum creatinine $>120 \mathrm{mmol} / \mathrm{l}$.

\section{Statistical analysis}

The years between January 1990 and September 2001 were divided into two 6 year periods 1990-1995 and 1996-2001. The number of eligible patients receiving AHT or LLA, or both, was assessed. Differences between the two time periods were examined.

Abbreviations: $A H T$, antihypertensive treatment; $C A D$, coronary artery disease; LLA, lipid lowering agent(s); SLE, systemic lupus erythematosus; SLEDAl, SLE Disease Activity Index 
Table 1 Characteristics of patients during two study intervals

\begin{tabular}{|c|c|c|}
\hline Characteristics & 1990-1995 & 1996-2001 \\
\hline No of patients & 559 & 576 \\
\hline Women & $495(88.6)$ & $511(88.7)$ \\
\hline \multicolumn{3}{|l|}{ Ethnicity } \\
\hline White & $450(80.5)$ & $432(75.0)$ \\
\hline Black & $45(8.1)$ & $48(8.3)$ \\
\hline Chinese & $39(7.0)$ & $44(7.6)$ \\
\hline Other & $25(4.5)$ & $52(9.0)$ \\
\hline Age at diagnosis, mean (SD) & $32.0(13)$ & $31.2(12.8)$ \\
\hline Age at entry to study, mean (SD) & $41.1(13.4)$ & 42.1 (14.2) \\
\hline Disease duration at entry, mean (SD) & $9.1(7.5)$ & $11.0(9.3)$ \\
\hline Number dead in interval & 31 & \\
\hline AMS during interval, mean (SD) & $4.7(3.6)$ & $5.15(4.1)$ \\
\hline Renal disease & $221(39.5)$ & $268(46.5)$ \\
\hline Nephrotic syndrome & $36(6.4)$ & $42(7.3)$ \\
\hline Renal insufficiency & $82(14.7)$ & $98(17.0)$ \\
\hline
\end{tabular}

\section{RESULTS}

Between 1990 and 1995, 559 patients were seen, whereas between 1996 and 2001576 patients were seen. The patient characteristics in these two periods were similar (table 1). Of the 559 patients seen in the first period, 204 (36\%) were hypertensive and 180 (88\%) were receiving AHT (table 2). Of the 576 patients seen in the second period, 241 (42\%) were hypertensive and 232 (96\%) of these were receiving AHT (table 2). Although the prevalence of hypertension was not statistically different between the two study periods, the treatment for hypertension was statistically significantly more often prescribed in the latter period. Moreover, between 1990 and 1995, 236 (42\%) had hypercholesterolaemia and $21(9 \%)$ were being treated with LLA, while between 1996 and 2001, 261 patients (45\%) had hypercholesterolaemia and 74 $(28 \%)$ were treated with LLA (table 2$)$. Although there was no difference in the prevalence of hyperlipidaemia between the two study periods, the use of LLA was significantly higher in the second period.

\section{DISCUSSION}

Our previous quality assurance study of patients followed up before 1996 showed that even in patients who subsequently developed an acute coronary event, previous treatment of risk factors such as hypertension and hyperlipidaemia was inadequate. The current study, which examined changes in trends of treatment of these risk factors in patients with SLE, shows that although there has been an improvement in treatment, a significant number of patients eligible for these treatments still remain untreated, especially the patients with hypercholesterolaemia. Despite the current more frequent use of statins, in general, and the increased knowledge of atherosclerosis in SLE, there were still only $28 \%$ of patients with hypercholesterolaemia being adequately managed.

The need to manage both hypercholesterolaemia and hypertension adequately in patients with SLE has been well established. Bruce et al divided 134 patients with SLE into three groups: those with normal cholesterol throughout the first 3 years of their disease, variable hypercholesterolaemia; those with intermittently raised cholesterol levels in the first 3 years, and sustained hypercholesterolaemia; and those with raised cholesterol levels in each of the first 3 years of their disease. ${ }^{11}$ Within that 3 year period, $25 \%$ were classified as normal cholesterol, $35 \%$ as variable hypercholesterolaemia, and $40 \%$ of patients as sustained hypercholesterolaemia. Over 10 years of follow up CAD events occurred in one patient (3\%) in the group with normal cholesterol, in three patients $(6.4 \%)$ in the group with variable hypercholesterolaemia, and
Table 2 Comparison of occurrence and treatment of hypertension and hyperlipidaemia in two study periods

\begin{tabular}{lccc}
\hline & \multicolumn{1}{l}{$1990-1995$} & $1996-2001$ & $\chi^{2}$ \\
\hline No of patients & 559 & 576 & \\
Hypertensive & 204 & 241 & 0.065 \\
Receiving anti-hypertensive & & & \\
treatment & 180 & 232 & 0.0013 \\
Hyperlipidaemia & 236 & 261 & 0.293 \\
Lipid lowering treatment & 21 & 74 & $<0.0001$ \\
\hline
\end{tabular}

in 15 patients $(28.7 \%)$ in the group with sustained hypercholesterolaemia. Moreover, several studies have found that in their patients with SLE, hypercholesterolaemia and hypertension were associated with CAD. ${ }^{2-4}$ Further, Rahman et al found that patients with SLE had fewer total CAD risk factors than patients with non-connective tissue disease with accelerated atherosclerosis. ${ }^{12}$ On this basis they speculated that predictive models (such as the Framingham risk factor calculation) are likely to underestimate the risk of CAD in this patient population.

The failure of physicians to rapidly adopt practices that would treat hypercholesterolaemia and hypertension in patients with SLE reflects a significant gap between published evidence from randomised control studies and clinical practice. Indeed, as Naylor remarked "clinical decisions do not always reflect even clear cut research evidence" ${ }^{\prime \prime}{ }^{13}$ referring to a study conducted by Majumdar et al which examined the speed with which academic physicians adopt new practices in response to evidence from randomised control studies. ${ }^{14}$

For hypertension and hypercholesterolaemia, the first large prospective study to identify them as risk factors was published in 1987, and two other large prospective studies followed in 1992 and 1997. ${ }^{2-4}$ Rahman et al showed that hypertension is a common occurrence in SLE and is associated with increased vascular events and mortality. ${ }^{5}$ The increase in vascular event in patients with hypertension is best explained by its association with hypercholesterolaemia. Additional evidence that identified hypertension and hypercholesterolaemia as risk factors for CAD in SLE, including carotid ultrasound studies and dual isotope myocardial perfusion imaging, was later published in the mid to late nineties. ${ }^{67}$ Despite the findings cited above, the present study found that between 1996 and 2001, only $28 \%$ of patients with hypercholesterolaemia were adequately managed. Over the entire 12 years of these observations, only $22 \%$ of patients with hypercholesterolaemia were adequately managed.

In the present study, hypertension was better managed than hypercholesterolaemia. This is similar to our previous findings. ${ }^{8}$ This is probably because blood pressure is measured at every clinic visit, whereas hypercholesterolaemia is a laboratory test done intermittently and not necessarily as part of a routine assessment.

In this study we found that $51 \%$ of patients with SLE had hypercholesterolaemia, which we defined as having at least one reading of random total cholesterol of $>6.2 \mathrm{mmol} / \mathrm{l}$. Differences in estimates of this disorder in SLE vary greatly, mainly owing to differences in defining "normal" cholesterol level. ${ }^{7}$ Using the same upper limit as our own, Manzi et al found that $6 \%$ of their patients had hypercholesterolaemia. ${ }^{4}$ In contrast, Bruce et al, using $5.2 \mathrm{mmol} / \mathrm{l}$ as the upper limit, found that $75.4 \%$ of their patients had hypercholesterolaemia within 3 years of diagnosis. ${ }^{8}$ Petri et al found that $56 \%$ of their patients had at least one reading of cholesterol $>5.2 \mathrm{mmol} / \mathrm{l}$ during a 1 year period. ${ }^{15}$ 
This study has several limitations. Firstly, it reflects the management strategies of a single centre and thus may not be generaliseable. Secondly, we limited our definition of the management of hypercholesterolaemia and hypertension to the administration, respectively, of LLA and anti-hypertensive drugs. We did not include lifestyle change as appropriate treatment, as this is not adequately recorded in our database. However, as Hearth-Holmes et al found, diet does not have a major effect on lipid values in SLE. ${ }^{16}$

Quality improvement is defined as "a continuous process that identifies problems in healthcare delivery, examines solutions to those problems, and regularly monitors solutions for improvement". Our first quality improvement study identified a problem with management of hypercholesterolaemia in patients who subsequently had coronary artery events. This knowledge was then incorporated into our routine clinical care in the Lupus Clinic. This quality improvement study measured the clinical response to abnormal biochemical risk factors over two periods of time with routine clinical care, without targeted education. The study demonstrates that although there was an improvement in care, management of hypercholesterolaemia is still not maximised. This study provides impetus for a specific education programme for our patients. Additional strategies to deal with these risk factors in lupus patients are currently underway.

\section{Authors' affiliations}

M B Urowitz, D D Gladman, D Ibanez, Y Berliner, Rheumatology Department, University Health Network, Toronto Western Hospital, Toronto, Canada

Correspondence to: Dr M B Urowitz, Rheumatology Department, University Health Network, Toronto Western Hospital, 399 Bathurst St, Toronto, ON M5T 2S8, Canada; m.urowitz@utoronto.ca

Accepted 19 June 2005

\section{REFERENCES}

1 Bruce IN, Gladman DD, Urowitz MB. Premature atherosclerosis in SLE. Rheum Dis Clin North Am 2000;26:257-78.

2 Gladman DD, Urowitz MB. Morbidity in systemic lupus erythematosus. J Rheumatol 1987; 14(suppl 13):223-6.

3 Petri M, Perez-Gutthan S, Spence D, Hochberg MC. Risk factors for coronary artery disease in patients with systemic lupus erythematosus. Am J Med 1992;93:513-19.

4 Manzi S, Meilahn EN, Rairie JE, Conte CG, Medsger TA Jr, JansenMcWilliams $L$, et al. Age-specific incidence of rates of myocardial infarction and angina in women with systemic lupus erythematosus: comparison with the Farmingham study. Am J Epidemiol 1997; 145:408-15.

5 Rahman P, Aguero S, Gladman DD, Hallett D, Urowitz MB. Vascular events in hypertensive patients with SLE. Lupus 2000;9:672-5.

6 Manzi, S, Selzer F, Sutton-Tyrrell K, Fitzgerald SG, Rairie JE, Tracy RP, et al. Prevalence and risk factors of carotid plaque in women with systemic lupus erythematosus. Arthritis Rheum 1999;42:51-60.

7 Bruce IN, Gladman DD, Ibanez D, Urowitz MB. Single photon emission computed tomography dual isotope myocardial perfusion imaging in women with systemic lupus erythematosus. II. Predictive factors for perfusion abnormalities. J Rheumatol 2003;30:288-91.

8 Bruce IN, Gladman DD, Urowitz MB. Detection and modification of risk factors for coronary artery disease in patients with systemic lupus erythematosus: a quality improvement study. Clin Exp Rheumatol 1998; 16:435-40.

9 Gladman DD, Ibañez D, Urowitz MB. SLE Disease Activity Index 2000 J Rheumatol 2002;29:288-91.

10 Ibanez D, Urowitz MB, Gladman DD. Summarizing disease features over time: I. Adjusted mean SLEDAI derivation and application to an index of disease activity in lupus. J Rheumatol 2003;30:1977-82.

11 Bruce IA, Urowitz MB, Gladman DD, Hallett DC. Natural history of hypercholesterolemia in systemic lupus erythematosus. J Rheumatol 1999;26:2137-43.

12 Rahman P, Urowitz MB, Gladman DD, Bruce IA, Genest J Jr. Contribution of tradidtional risk factors to coronary artery disease in patients with systemic lupus erythmatosus. J Rheumatol 1999;26:2363-8.

13 Naylor CD. Putting evidence into practice. Am J Med 2002;113:161-3.

14 Majumdar SR, Wei-Ching C, Armstrong PW. Do the investigative sites that take part in a positive clinical trial translate that evidence into practice? Am J Med 2002;113:140-5.

15 Petri M, Spence D, Bone LR, Hochber MC. Coronary artery disease risk factors in the John Hopkins Lupus Cohort: prevalence, recognition by patients and preventative practices. Medicine (Baltimore) 1992;71:291-302.

16 Hearth-Holmes M, Baethge BA, Broadwell L, Wolf RE. Dietary treatement of hyperlipidemia in patients with systemic lupus erythematosus. J Rheumatol $1995 ; 2: 450-4$ 